\title{
Transformation of COUPY fluorophores into a novel class of visible light-cleavable photolabile protecting groups
}

\author{
Marta López-Corrales, ${ }^{[a]}$ Anna Rovira, ${ }^{[a]}$ Albert Gandioso, ${ }^{[a]}$ Manel Bosch, ${ }^{[b]}$ Santi Nonell, ${ }^{[c]}$ and Vicente \\ Marchán*[a] \\ [a] M. López-Corrales, A. Rovira, Dr. A. Gandioso, Dr. V. Marchán \\ Departament de Química Inorgànica i Orgànica \\ Secció de Química Orgànica, IBUB, Universitat de Barcelona \\ Martí i Franquès 1-11, E-08028 Barcelona (Spain) \\ E-mail: vmarchan@ub.edu \\ [b] Dr. M. Bosch \\ Unitat de Microscòpia Òptica Avançada \\ Centres Científics i Tecnològics, Universitat de Barcelona \\ Av. Diagonal, 643, E-08028 Barcelona (Spain) \\ [c] Prof. S. Nonel \\ Institut Químic de Sarrià, Universitat Ramon Llull \\ Vía Augusta 390, E-08017 Barcelona (Spain)
}

Supporting information for this article is given via a link at the end of the document

\begin{abstract}
Although photolabile protecting groups (PPGs) have found widespread applications in several fields of chemistry, biology and materials science, there is a growing interest in expanding the photochemical toolbox to overcome some of the limitations of classical caging groups. In this work, the synthesis of a new class of visible-light-sensitive PPGs based on low-molecular weight COUPY fluorophores with several attractive properties, including longwavelength absorption, is reported. Besides being stable to spontaneous hydrolysis in the dark, COUPY-based PPGs can be efficiently photoactivated with yellow $(560 \mathrm{~nm})$ and red light $(620 \mathrm{~nm})$ under physicological-like conditions, thereby offering the possibility of unmasking functional groups from COUPY photocages under irradiation conditions in which other PPGs remain stable. Additionally, COUPY photocages exhibit excellent cellular uptake and accumulate selectively in mitochondria, opening the door to delivering caged analogues of biologically active compounds into this organelle.
\end{abstract}

Photolabile protecting groups (PPGs; also commonly termed photoremovable protecting groups or caging groups) have found widespread applications in several fields of chemistry, biology and materials manufacturing owing to the extraordinary properties of light. $^{[1]}$ Indeed, suitable light irradiation can be delivered with high spatiotemporal precision to control molecular processes without causing contamination of samples. This is particularly important in the case of caged analogues of biologically active compounds (e.g., biomolecules, neurotransmitters, antibiotics, anticancer agents, etc.), since unmasking of key functionalities can be triggered without using any chemical reagent. ${ }^{[2]}$ Besides orthogonality, in vivo applications require the use of PPGs with long-wavelength absorption to achieve deeper tissue penetration and to avoid the phototoxicity associated with short wavelengths such as UV and blue light. ${ }^{[3]}$

The development of novel PPGs that can be efficiently photoactivated with visible and near-IR light has raised a growing interest to expand the photochemical toolbox in biological applications, ${ }^{[2]}$ as well as to facilitate the design of complex wavelength-selective uncaging systems in which two or more PPGs can be removed sequentially from a single compound. ${ }^{[1 d]}$ Until recently, most caging groups described in the literature with photoactivation capability by visible light are based on classical and non-classical organic chromophores (e.g., o-nitrobenzyl, ${ }^{[4]}$ coumarin, ${ }^{[5]}$ BODIPY, ${ }^{[6]}$ cyanine ${ }^{[7]}$ and quinone ${ }^{[8]}$ derivatives) or on metal complexes (e.g., ruthenium polypyridyl). ${ }^{\left[{ }^{[]}\right.}$Besides long-wavelength excitation capability, an ideal PPG is expected to fulfill certain additional requirements such as high photolytic efficiency (which requires either large molar absorption coefficients at the absorption maxima or high uncaging quantum yields, or both), aqueous solubility and dark stability to spontaneous hydrolysis under physiological conditions. The structural complexity of the caging chromophore, molecular weight, synthetic accessibility and conjugatability are also key parameters to be considered when designing new caging groups.

Coumarin-based caging groups fulfill some of the criteria for an ideal PPG owing to the privileged architecture of the coumarin scaffold, which allows the attachment of the compound to be caged through different types of bonds (e.g., ester, carbamate or carbonate). In addition, the photophysical and photochemical properties of coumarin-based PPGs can be fine-tuned through smart structural modifications such as by replacing electrondonating $\mathrm{N}, \mathrm{N}$-dialkylamino groups at position 7 on the wellknown (coumarin 4-yl)methyl skeleton by azetidine. ${ }^{[10]}$ The modification of the carbonyl group of the electron-withdrawing lactone moiety in conventional DEACM or DEACE caging groups (Scheme 1) has also been used to increase the electronic delocalization along the $\pi$-conjugated system, ${ }^{[5 b d e f]}$ dicyanomethylenecoumarin derivatives being particularly attractive as they exhibit an absorption maxima around $500 \mathrm{~nm}$ which allows uncaging with green light. ${ }^{[5 \mathrm{de}]}$ So far, we have synthesized a series of dicyanocoumarinylmethyl (DEAdcCM)and dicyanocoumarinylethyl (DEAdcCE)-based photocages of 
carboxylic acids (Scheme 1) and demonstrated a faster photolysis rate in the DEAdcCE series, which is a consequence of the higher stability of the secondary carbocation intermediate generated upon the solvent-assisted photoheterolytic ester bond cleavage..$^{[5 d e]}$ A similar behaviour was found in coumarin analogues in which one of the cyano groups in DEAdcCM and DEAdcCE was replaced with $p$-nitrophenyl. ${ }^{[5]}$

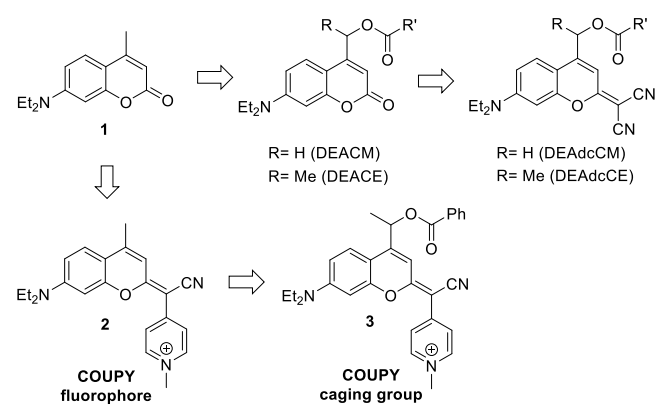

Scheme 1. Previously reported coumarin- and dicyanocoumarin-based PPGs and transformation of COUPY fluorophore $\mathbf{2}$ into a novel caging group synthesized in this work.

With the aim of further red-shifting the absorption and emission maxima of the conventional coumarin scaffold, we have recently developed a novel class of coumarin-based fluorophores, COUPYs, in which the carbonyl function in coumarin 1 was replaced with cyano( $\mathrm{N}$-alkyl-4-pyridinium)-methylene moieties (e.g., compound 2 in Scheme 1). ${ }^{[11]}$ Due to a higher push-pull character compared with dicyanomethylene coumarins, COUPY dyes exhibit several interesting photophysical properties such as far-red to NIR emission, large Stokes' shifts and high photostability. ${ }^{[11]}$ Conjugatable versions of COUPY fluorophores can also be obtained through $\mathrm{N}$-alkylation of the pyridine heterocycle. ${ }^{[12]}$ On the basis of these precedents, we envisaged the transformation of COUPY fluorophores into novel coumarinbased PPGs for visible-light uncaging. As shown in Scheme 1, here we focused on the synthesis of the first COUPY photocage (compound 3) in which benzoic acid was chosen as a model cargo and caged through the formation of an ester bond. Taking into account that the rate of the overall uncaging process in coumarin-based PPGs depends on the rate constant of the initial heterolytic C-O bond cleavage, ${ }^{[13]}$ we decided to incorporate a methyl group in the position adjacent to this bond in the coumarin skeleton to stabilize the carbocation intermediate and increase the photodeprotection rate..$^{[5 \operatorname{def}]}$

Compound $\mathbf{3}$ was efficiently synthesized in two steps from thiocoumarin 4, which was previously obtained from commercially available coumarin $\mathbf{1}$ in four steps (Scheme 2). ${ }^{[5 e f]}$ First, condensation of $\mathbf{4}$ with 4-pyridylacetonitrile in the presence of $\mathrm{NaH}$ followed by treatment with silver nitrate afforded COUPY scaffold $\mathbf{5}$ with high yield (87\%) after purification by silica column chromatography. After $\mathrm{N}$-methylation of the pyridine heterocycle with methyl trifluoromethanesulfonate, the target COUPY-caged model compound $\mathbf{3}$ was obtained as a pink solid with excellent yield $(98 \%)$ and fully characterized by HR ESI-MS and NMR $\left({ }^{1} \mathrm{H}\right.$, ${ }^{13} \mathrm{C}$, and ${ }^{19} \mathrm{~F}$ ). As previously found with some COUPY fluorophores, ${ }^{[11]}$ the ${ }^{1} \mathrm{H}$ NMR spectrum of $\mathbf{5}$ showed two sets of proton signals in a $\sim 85: 15$ ratio, which accounts for the existence of two interconverting rotamers around the exocyclic
$\mathrm{C}=\mathrm{C}$ bond. Full NMR characterization by using 2D COSY and NOESY experiments (Figure S1) allowed to conclude that the $E$ rotamer (as usually drawn in the manuscript) was the major species in solution. By contrast, only the $E$ rotamer was present in solution in the case of COUPY-caged compound $\mathbf{3}$ according to $1 \mathrm{D}$ and 2D NMR spectra (Figure S2), which parallels the behavior of $\mathrm{N}$-methylated COUPY dyes. ${ }^{[1]]}$

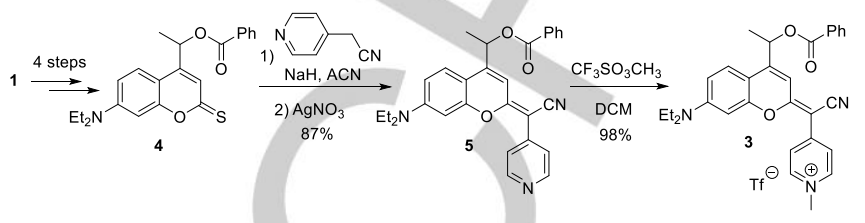

Scheme 2. Synthesis of COUPY-caged model compound 3.

The photophysical properties of compound $\mathbf{3}$ were investigated in a 1:1 (v/v) mixture of PBS buffer and ACN (Table S1) and compared with those of the parent dye (2). As shown in Figure 1, compound $\mathbf{3}$ exhibited an intense, relatively narrow absorption band in the visible spectrum, with an absorption maximum centered at $557 \mathrm{~nm}$, slightly extended into the red-light region and red-shifted with respect to coumarin 2 (ca $11 \mathrm{~nm}$ ). This bathochromic effect was more pronounced for the emission wavelength ( $\lambda_{\mathrm{em}}=619 \mathrm{~nm}$ in $3 \mathrm{vs} \lambda_{\mathrm{em}}=603 \mathrm{~nm}$ in 2) and, as a result, the Stokes' shift was larger in $\mathbf{3}(62 \mathrm{~nm})$ than in the parent fluorophore $(57 \mathrm{~nm})$. By contrast, the fluorescent quantum yield was slightly reduced in the caged compound (e.g., $\Phi_{F}=0.10$ in 3 vs. $\Phi_{F}=0.22$ in 2). In addition, COUPY photocage exhibited a relatively large molar absorption coefficient $\left(38,000 \mathrm{M}^{-1} \mathrm{~cm}^{-1}\right)$.
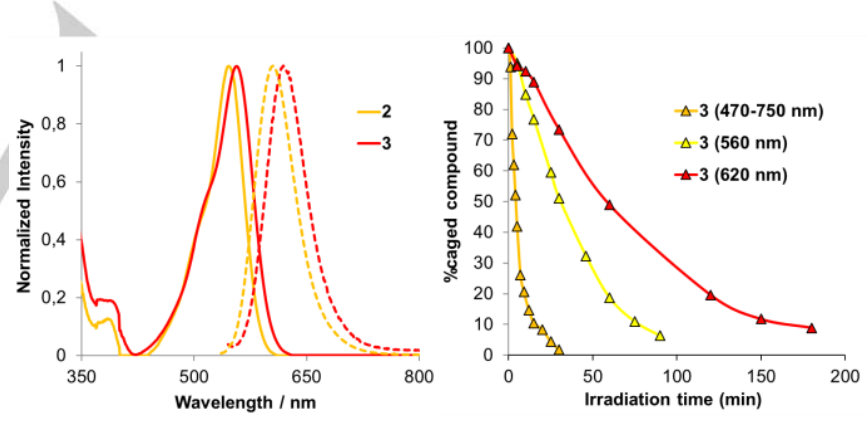

Figure 1. Left: comparison of the absorption (solid lines) and fluorescence (dotted lines) spectra of COUPY-caged model compound $\mathbf{3}$ with those of the parent COUPY dye (2). Right: plot of the temporal evolution of the amount of 3 after irradiation with visible LED light. The lines connecting the experimental points are meant to aid the reader in visualizing the data. All the experiments were performed in a 1:1 (v/v) mixture of PBS buffer and ACN.

The photoactivation of COUPY-caged model compound 3 was first evaluated in PBS/ACN $1: 1$ at $37^{\circ} \mathrm{C}$ under irradiation with a LED source covering from the cyan to the red region of the visible spectrum (470-750 nm; $150 \mathrm{~mW} \mathrm{~cm}^{-2}$; see Figure S3). The course of the uncaging process was monitored by reversedphase HPLC-MS in terms of the consumption of the starting caged compound with irradiation time (Figure S4). As shown in Figure 1, the concentration of $\mathbf{3}$ decreased gradually with irradiation time. Photoactivation of $\mathbf{3}$ proceeded in a clean fashion and gave a main photolytic product (coumarin alcohol 6) 
and a minor vinyl coumarin derivative (7), the latter being formed through a $\beta$-elimination reaction from the secondary carbocation intermediate generated upon photoheterolysis of the ester bond (Scheme 3). Photolysis of 3 was almost complete (ca $90 \%$ ) after $15 \mathrm{~min}$ of irradiation with the wide-band LED $\left(k_{\mathrm{u}}=0.172\right.$ $\left.\mathrm{min}^{-1}\right)$, whereas it was required more than 90 min to completely uncage it with the same LED equipped with a $560 \mathrm{~nm}$ bandpass filter (yellow light, $40 \mathrm{~mW} \mathrm{~cm}{ }^{-2}, k_{\mathrm{u}}=0.031 \mathrm{~min}^{-1}$; see Figure 1 and Figures S3 and S5), which can be attributed to the reduced irradiance. Importantly, $\mathbf{3}$ was found completely stable after incubation for $2 \mathrm{~h}$ at $37^{\circ} \mathrm{C}$ in the dark under the irradiation conditions. In addition, the uncaging of benzoic acid from compound 3 and the release of coumarin alcohol 6 was also confirmed by monitoring the photolysis process by ${ }^{1} \mathrm{H}$ NMR in a mixture of DMSO- $d_{6}$ and $\mathrm{D}_{2} \mathrm{O}$ (Figure 2).

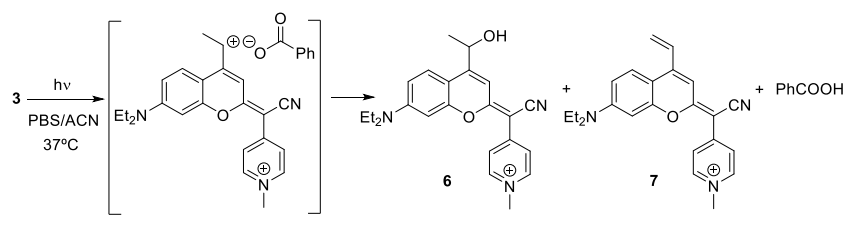

Scheme 3. Mechanistic interpretation of the photolysis process of compound 3 under visible light irradiation.

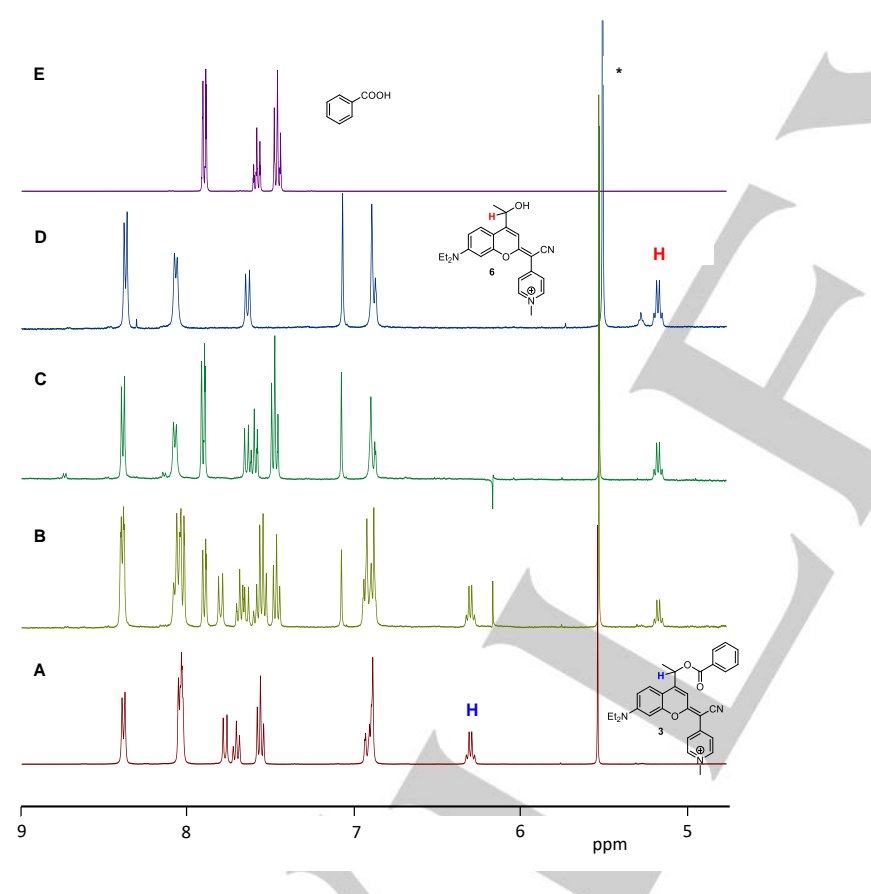

Figure 2. Expansion of the ${ }^{1} \mathrm{H}$ NMR spectrum of 3 in DMSO- $d_{6} / D_{2} \mathrm{O} 7: 3(\mathrm{v} / \mathrm{v})$ $(400 \mathrm{MHz}$ ) before irradiation $(\mathrm{A})$ and after 30 -min $(B)$ and 2-h $(\mathrm{C})$ photolysis at $37^{\circ} \mathrm{C}$ with visible light (470-750 $\mathrm{nm}$ range), and of coumarin 6 (D) and benzoic acid (E). 'DCM traces.

Encouraged by these results and taking into account the significant absorptivity of the COUPY chromophore around 600 $\mathrm{nm}$, we decided to investigate the photosensitivity of compound 3 under red light irradiation. To our delight, the concentration of 3 decreased gradually with time upon irradiation with a $620 \mathrm{~nm}$ LED source (130 $\mathrm{mW} \mathrm{cm}^{-2}$, Figures 1 and S6) although, as expected on the basis of the overlap between the LED sources and the absorption spectra (Figure S7), 3 took longer to react on irradiation with red light as compared to yellow light, due to the lower rate of light absorption (about $50 \%$ of $\mathbf{3}$ was uncaged after irradiation for $1 \mathrm{~h}$ ). The actual rate of light absorption under the three LEDs is shown in Figure S8. It is worth noting that the same photolytic products (6 and 7) were observed both with yellow and red light (Scheme 3 ).

Using the data in Figure S8 and the initial rate of deprotection of compound 3, the quantum yield ( $\phi$ Phot $)$ for deprotection could be calculated and similar values were found for both the yellow and red LEDs (6.0 × $10^{-5}$ and $5.0 \times 10^{-5}$, respectively; Figure S9), confirming that the differences in the rates of deprotection actually reflect the relative rates of light absorption. The photolytic efficiency of the uncaging process is then much higher with yellow light due to the large molar absorptivity of the compound at $560 \mathrm{~nm}$ (Table S2). It is worth noting that the quantum yields are higher than that previously reported by us for a coumarin-caged compound containing $p$-nitrophenyl moiety instead of $\mathrm{N}$-methylpyridinium, ${ }^{[5 f]}$ and of the same order than other visible-light-sensitive photoprotecting groups, including a green-light sensitive BODIPY derivative recently described by Szymanski and collaborators (ca $\left.4 \times 10^{-5}\right)$. ${ }^{66]}$

Next, we investigated if COUPY caging groups could be applied in the design of selective uncaging systems. ${ }^{[1 e]}$ Two main approaches have been described to control two or more molecular events with light. On the one hand, we ${ }^{[5 e]}$ and others, including Heckel's group, ${ }^{[14]}$ have demonstrated that selective uncaging can be achieved through irradiation with a single wavelength of light by exploiting the differences in deprotection kinetics of structurally-related PPGs (e.g., with green light by using DEAdcCM and DEAdcCE-based caging groups) when attached to the molecule to be caged through a different atom or photocleavable bond. ${ }^{[5 e]}$ On the other hand, the use of two or more PPGs with significantly different spectral and/or photochemical properties $\left(\lambda_{\max }, \varepsilon, \phi_{\text {Phot }}\right)$ can also be exploited to sequentially unmask several functionalities with different wavelengths of light.

On the basis of the photolysis studies with compound $\mathbf{3}$, we wondered if sequential uncaging of two coumarin-protected functionalities in the same reaction mixture could be achieved by sequentially irradiating with biologically-compatible visible light. For this purpose, an equimolar mixture of COUPY-caged compound $\mathbf{3}$ and DEAdcCE-caged compound $\mathbf{8}^{[5 \mathrm{e}]}$ (Scheme 4) was irradiated first with green light $(505 \mathrm{~nm})$. Although dicyanocoumarin 8 was completely uncaged after $15 \mathrm{~min}$, about $75 \%$ of compound 3 was also deprotected (Figure S10), which is not surprising given its substantial absorbance at $505 \mathrm{~nm}$. However, by irradiating first with red light $(620 \mathrm{~nm}), 3$ was completely uncaged within $2.5 \mathrm{~h}$ while 8 remained stable until irradiation with green light was carried out (Figure S11). In both cases, the formation of the expected photolytic coumarins ( 6 and 9) was confirmed by MS. This experiment demonstrated that the sequential uncaging with visible light of the same functional group (a carboxylic acid in our models) from two different caged compounds can be achieved by combining COUPY caging groups with other coumarin-based PPGs. 


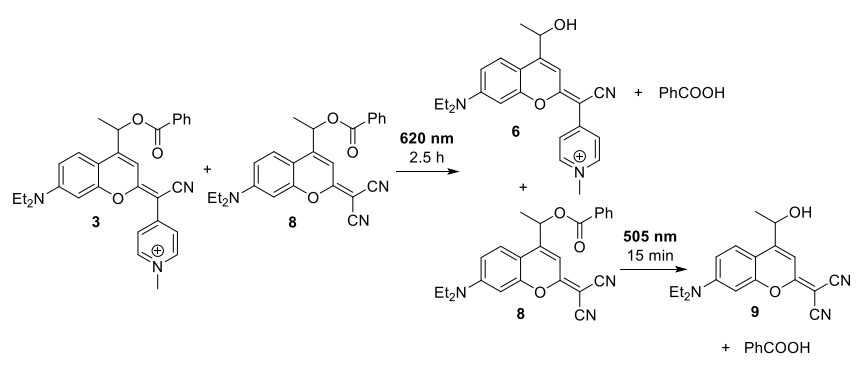

Scheme 4. Sequential uncaging of compounds 3 and 8 by sequential irradiating with red $(2.5 \mathrm{~h})$ and green $(15 \mathrm{~min})$ light.

Finally, the cellular uptake and organelle localization of COUPYcaged model compound $\mathbf{3}$ in living HeLa cells were investigated by using confocal microscopy. As shown in Figure 3 , the fluorescence signal after excitation with a yellow light laser $\left(\lambda_{\mathrm{ex}}=\right.$ $561 \mathrm{~nm}$ ) was clearly observed inside the cells after only $30 \mathrm{~min}$ of incubation $(1 \mu \mathrm{M})$ owing to the great brightness of the COUPY chromophore, and the overall pattern of staining suggested accumulation in mitochondria, and, to a lesser extent, in intracellular vesicles. In addition, no cell toxicity was observed during these studies. These observations confirmed that the incorporation of a small cargo at the 4-position of the coumarin skeleton (benzoic acid) does not negatively influences neither the good cellular uptake nor organelle selectivity of the parent COUPY fluorophores. ${ }^{[11]}$ Indeed, co-localization experiments using two commercially available specific markers for labeling mitochondria (MitoTracker Green FM, MTG) and lysosomes (Lysotracker Green FM, LTG) confirmed the observed subcellular localization. In the case of mitochondria (Figure 4, AC), a Pearson's sample correlation coefficient of $0.85 \pm 0.05$ was obtained indicating a good correlation between $\mathbf{3}$ and MTG. Manders' colocalization coefficients were also measured and showed that $68 \% \pm 0.12$ of compound 3 staining colocalized with MTG and that $90 \% \pm 0.08$ of MTG colocalized with compound 3 signal. On the other hand, co-localization experiments with LTG (Figure 4, D-F) confirmed that most of fluorescence observed in intracellular vesicles along the cytoplasm was associated with lysosome accumulation. Pearson's coefficient was $0.59 \pm 0.07$ and Mander's coefficients were $4 \% \pm 0.04$ (compound 3 colocalizing with LTG) and $80 \% \pm 0.24$ (LTG colocalizing with 3).

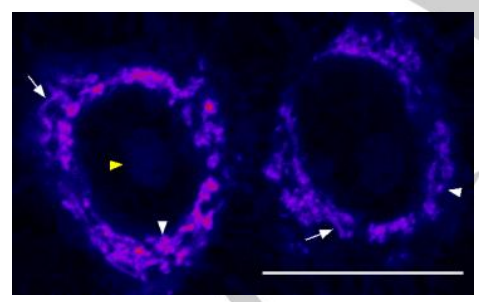

Figure 3. Confocal section of HeLa cells stained with compound 3. Arrows point out mitochondria, white arrowheads point out vesicles and yellow arrowheads point out the nucleoli. LUT $=$ Fire. Scale bar $=20 \mu \mathrm{m}$.
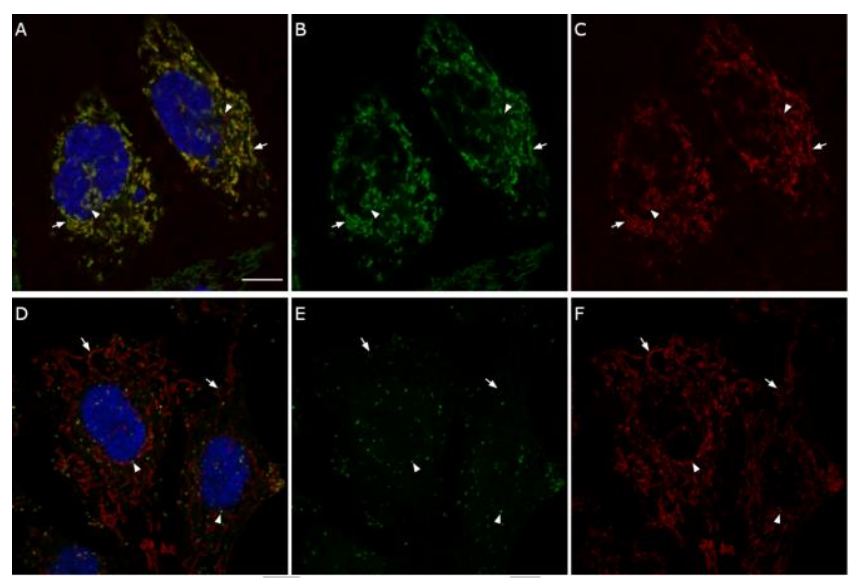

Figure 4. Co-localization studies with compound $\mathbf{3}$ and Mitotracker Green FM (A-C) or Lysotracker Green FM (D-F). Single confocal plane of HeLa cells incubated with $3(1 \mu \mathrm{M}$, red) and Mitotracker Green FM $(0.1 \mu \mathrm{M}$, green) or Lysotracker Green FM (0.2 $\mu \mathrm{M}$, green). A), D) Overlay of the two staining. C), F) compound 3, B) Mitotracker Green FM signal. E) Lysotracker Green FM staining. White arrows point out mitochondria and white arrowheads point out coumarin 3 vesicles. Scale bar: $10 \mu \mathrm{m}$. All images are at the same scale than A.

In summary, we have developed a new class of visible-lightsensitive PPGs based on COUPY fluorophores for the protection of carboxylic acids, which exhibit several attractive properties for application in biological systems, such as long wavelength absorption owing to a large $\pi$-conjugated system, large molar extinction coefficient and high deprotection rate in aqueous media. This novel caging group is completely stable to spontaneous hydrolysis in the dark but can be efficiently photoactivated by green- (505 nm), yellow- $(560 \mathrm{~nm})$ and redLED light $(620 \mathrm{~nm})$ under physicological-like conditions (PBS buffer, $37^{\circ} \mathrm{C}$ ), proceeding in a clean fashion as confirmed both by HPLC-MS and NMR analysis. COUPY caging groups presented here will complement the photochemical toolbox of known coumarin-based PPGs, since they offer the possibility of unmasking functional groups from COUPY photocages under visible light irradiation conditions (e.g., with yellow/red light) in which other conventional (e.g., DEACM/DEACE) and nonconventional (e.g., DEAdcCE/DEAdcCE) coumarin-based caging groups remain stable. Importantly, the COUPY-caged model compound described here exhibited an excellent cellular uptake and accumulated selectively in mitochondria owing to the $\mathrm{N}$-alkylpyridinium-based structure of COUPY chromophore, which opens the door to delivering biologically-active compounds selectively into this key organelle..$^{[5 i, 6 \mathrm{~d}]}$ Work is in progress to further red-shift the absorption maxima of COUPYbased caging groups to the phototherapeutic window region (650-900 nm), as well as to improve the deprotection efficiency through additional chemical modifications in the COUPY chromophore.

\section{Acknowledgements}

This work was supported by funds from the Spanish Government (MCIU/AEI/FEDER, UE; grants CTQ2017-84779-R and CTQ2016-78454-C2-1-R) and the Generalitat de Catalunya (2017 DI 072). The authors acknowledge helpful assistance of 
Dr. Francisco Cárdenas (NMR) and Dr. Irene Fernández and Laura Ortiz (MS) from CCiTUB, and of Arturo Prudencio (Microbeam, Spain) in the development of the irradiation device. A.R. and A.G. were recipient fellows of the University of Barcelona.

Keywords: coumarin $\cdot$ protecting groups $\cdot$ caged compounds • photolysis $\cdot$ mitochondria

[1] a) G. Mayer, A. Heckel, Angew. Chem. Int. Ed. 2006, 45, 4900-4921; b) C. Brieke, F. Rohrbach, A. Gottschalk, G. Mayer, A. Heckel, Angew. Chem. Int. Ed., 2012, 51, 8446-8476; c) P. Klán, T. Solomek, C. G. Bochet, A. Blanc, R. Givens, M. Rubina, V. Popik, A. Kostikov, J. Wirz, Chem. Rev., 2013, 113, 119-191; d) W. A. Velema, W. Szymanski, B. L. Feringa, J. Am. Chem. Soc., 2014, 136, 2178-2191; e) M. J. Hansen, W. A. Velema, M. M. Lerch, W. Szymanski, B. L. Feringa, Chem. Soc. Rev., 2015, 44, 3358-3377; f) A. Bardhan, A. Deiters, Curr. Opin. Str. Biol., 2019, 57, 164-175.

[2] J. M. Silva, E. Silva, R. L. Reis, J. Control. Release, 2019, 298, 154-176.

[3] a) K. Kalka, H. Merk, H. Mukhtar, J. Am. Acad. Dermatol., 2000, 42, 389-413; b) S. L. Jacques, S. L, Phys. Med. Biol., 2013, 58, R37-R61.

[4] H. K. Agarwal, R. Janicek, S.-H. Chi, J. W. Perry, E. Niggli, G. C. R. Ellis-Davies, J. Am. Chem. Soc., 2016, 138, 3687-3693.

[5] a) C. Menge, A. Heckel, Org. Lett., 2011, 13, 4620-4623; b) L. Fournier, I. Aujard, T. Le Saux, S. Maurin, S. Beaupierre, J.-B. Baudin, L. Jullien, Chem. Eur. J., 2013, 19, 17494-17507; c) J. P. Olson, H.-B. Kwon, K. T. Takasaki, C. Q. Chiu, M. J. Higley, B. L. Sabatini, G. C. R. EllisDavies, J. Am. Chem. Soc., 2013, 135, 5954-5957; d) A. Gandioso, M. Cano, A. Massaguer, V. Marchán, J. Org. Chem., 2016, 81, 11556-11564; e) A. Gandioso, M. Palau, A. Nin-Hill, I. Melnyk, C Rovira, S. Nonell, D. Velasco, J. García-Amorós, V. Marchán, ChemistryOpen, 2017, 6, 375-384; f) A. Gandioso, S. Contreras, I. Melnyk, J. Oliva, S. Nonell, D. Velasco, J. García-Amorós, V. Marchán, J. Org. Chem., 2017, 82, 5398-5408; g) Y. Chitose, M. Abe, K. Furukawa, J.-Y. Lin, T.-C. Lin, C. Katan, Org. Lett., 2017, 19, 2622-2625; h) Q. Lin, L. Yang, Z. Wang, Y. Hua, D. Zhang, B. Bao, C Bao, X. Gong, L. Zhu, Angew. Chem. Int. Ed., 2018, 57, 3722-3726; i) N. Wagner, M. Stephan, D. Höglinger, A. Nadler, Angew. Chem. Int Ed., 2018, 57, 13339-13343; j) M. Klausen, V. Dubois, G. Clermont, C Tonnelé, F. Castet, M. Blanchard-Desce, Chem. Sci., 2019, 10 4209-4219; k) M. Bojtár, A. Kormos, K. Kis-Petik, M. Kellermayer, P. Kele, Org. Lett. 2019, 21, 9410-9414.

[6] a) T. Slanina, P. Shrestha, E. Palao, D. Kand, J. A. Peterson, A. S. Dutton, N. Rubinstein, R. Weinstain, A. H. Winter, P. Klán, J. Am. Chem. Soc. 2017, 139, 15168-15175; b) K. Sitkowska, B. L. Feringa, W. Szymanski, J. Org. Chem., 2018, 83, 1819-1827; c) J. A. Peterson, C. Wijesooriya, E. J. Gehrmann, K. M. Mahoney, P. P. Goswami, T. R. Albright, A. Syed, A. S. Dutton, E. A. Smith, A. H. Winter, J. Am. Chem. Soc., 2018, 140, 7343-7346; d) D. Kand, L. Pizarro, I. Angel, A. Avni, D. Friedmann-Morvinski, R. Weinstain, Angew. Chem. Int. Ed., 2019, 58, 4659-4663.

[7] a) R. R. Nani, A. P. Gorka, T. Nagaya, H. Kobayashi, M. J. Schnermann, Angew. Chem. Int. Ed., 2015, 54, 13635-13638; R. R. Nani, A. P. Gorka, T. Nagaya, T. Yamamoto, J. Ivanic, H. Kobayashi, M. J. Schnermann, ACS Cent. Sci., 2017, 3, 329-337.

[8] D. P. Walton, D. A. Dougherty, J. Am. Chem. Soc., 2017, 139, 46554658.

[9] (a) M. Salierno, E. Marceca, D. S. Peterka, R. Yuste, J. Inorg. Biochem., 2010, 104, 418-422; b) J. Mosquera, M. I. Sánchez, J. L. Mascareñas, M. E. Vázquez, Chem. Comm., 2015, 51, 5501-5504; c) T. Voelker, E. Meggers, Curr. Opin. Chem. Biol., 2015, 25, 48-54; d) A. Li, C. Turro, J. Kodanko, Acc. Chem. Res., 2018, 51, 1415-1421; e) A. Li, C. Turro, J. J. Kodanko, Chem. Commun. 2018, 54, 1280-1290.

[10] G. Bassolino, C. Nançoz, Z. Thiel, E. Bois, E. Vauthey, P. RiveraFuentes. Chem. Sci. 2018, 9, 387-391.
[11] a) A. Gandioso, R. Bresolí-Obach, A. Nin-Hill, M. Bosch, M. Palau, A Galindo, S. Contreras, A. Rovira, C. Rovira, S. Nonell, V. Marchán, J Org. Chem., 2018, 83, 1185-1195; b) A. Gandioso, M. Palau, R. Bresolí-Obach, A. Galindo, A. Rovira, M. Bosch, S. Nonell, V. Marchán J. Org. Chem., 2018, 83, 11519-11531; c) A. Rovira, M. Pujals, A. Gandioso, M. López-Corrales, M. Bosch, V. Marchán, J. Org. Chem., 2020, 85, 6086-6097.

[12] a) A. Rovira, A. Gandioso, M. Goñalons, A. Galindo, A. Massaguer, M. Bosch, V. Marchán, J. Org. Chem., 2019, 84, 1808-1817; b) V. Novohradsky, A. Rovira, C. Hally, A. Galindo, G. Vigueras, A. Gandioso, M. Svitelova, R. Bresolí-Obach, H. Kostrhunova, L. Markova, J. Kasparkova, S. Nonell, J. Ruiz, V. Brabec, V. Marchán, Angew. Chem. Int. Ed., 2019, 58, 6311-6315.

[13] T. Eckardt, V. Hagen, B. Schade, R. Schmidt, C. Schweitzer, J. Bendig, J. Org. Chem., 2002, 67, 703-710.

[14] A. Rodrigues-Correia, D. Knapp-Behle, J. W. Engels, A. Heckel, Org. Lett., 2014, 16, 5128-5131. 


\section{Entry for the Table of Contents}

Insert graphic for Table of Contents here.

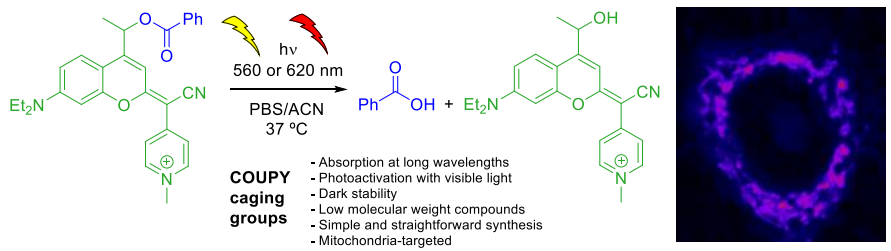

Insert text for Table of Contents here.

We report here the transformation of coumarin-based COUPY fluorophores into a new class of visible light-sensitive photolabile protecting groups which expand the current photochemical toolbox. Besides exhibiting an excellent cellular uptake, COUPY photocages accumulate selectively in mitochondria, thereby offering the possibility of delivering biologically active compounds within this organelle in a selective manner.

Institute and/or researcher Twitter usernames: University of Barcelona 